\title{
Einschleppung von Tandonia kusceri (Pulmonata: Milacidae) nach Transkarpatien und Verbreitung von Tandonia-Arten in der Ukraine
}

\author{
Introduction of Tandonia kusceri (Pulmonata: Milacidae) in Transcarpathia and \\ distribution of Tandonia species in Ukraine
}

\author{
Nina Gural-Sverlova ${ }^{1}$, Vasyl Gleba ${ }^{2} \&$ Roman Gural ${ }^{1}$ \\ ${ }^{1}$ State Museum of Natural History, National Academy of Sciences of Ukraine, Teatralna 18, UA-79008 Lviv, Ukraine, \\ e-mail: sverlova@pip-mollusca.org (corresponding author), (1) http://orcid.org/0000-0002-3892-5338, \\ (D) http://orcid.org/0000-0002-1546-1956 \\ ${ }^{2}$ Ukrainian Society for the Protection of Birds, UA-90332 Korolevo,Ukraine, e-mail: glebasileus@mail.ru
}

\begin{abstract}
Gural-Sverlova N., Gleba V. \& Gural R., 2019: Einschleppung von Tandonia kusceri (Pulmonata: Milacidae) nach Transkarpatien und Verbreitung von Tandonia-Arten in der Ukraine [Introduction of Tandonia kusceri (Pulmonata: Milacidae) in Transcarpathia and distribution of Tandonia species in Ukraine]. - Malacologica Bohemoslovaca, 18: 19-26. Online serial at <http://mollusca.sav.sk> 16-Nov-2019.
\end{abstract}

\begin{abstract}
Die eingeschleppte balkanische Nacktschnecke Tandonia kusceri wurde zum ersten Mal im Verwaltungsgebiet Transkarpatien im Westen der Ukraine nachgewiesen. Die Nacktschnecken wurden im Herbst 2018 am Rande der Siedlung Korolevo, Bezirk Vinogradov, unweit vom Eisenbahndepot gefunden. Die örtliche Bevölkerung nutzt diesen Standort als Deponie für Bauschutt. Bisher waren nur vereinzelte Fundorte von T. kusceri in der Südukraine (Verwaltungsgebiete Odessa und Nikolaev sowie die Krim) bekannt. Die ersten bekannten Exemplare dieser Art wurden in der Stadt Odessa im Jahre 1902 gesammelt und dann als Amalia rossica beschrieben. In der Ukraine kommen insgesamt drei Arten der Gattung Tandonia vor, deren Verbreitung auf einer Karte dargestellt wird. Tandonia kaleniczenkoi ist nur in der Bergkrim verbreitet. Tandonia cristata kommt vereinzelt auch in der Steppenzone der Ukraine vor. Außerdem ist ein Fundort dieser Art in Transkarpatien am Rand der Stadt Uzhgorod bekannt.
\end{abstract}

The introduced Balkan slug Tandonia kusceri was for the first time recorded in the Transcarpathian region in the west of Ukraine. The slugs were discovered in the autumn of 2018 at the outskirts of Korolevo settlement, Vinogradov district, near the railway depot. Local residents use this site as a dump for construction waste. Until now, only a few occurrences of T. kusceri were known from the south of Ukraine (Odessa and Nikolaev regions, Crimea). The first known specimens of this species were collected in Odessa city in 1902 and subsequently described as Amalia rossica. In total, three species of Tandonia occur in Ukraine, the known localities of which are shown on a map. Tandonia kaleniczenkoi occurs only in the Mountainous Crimea. Tandonia cristata is also sporadically found in the steppe zone of Ukraine, one finding of this species in the Transcarpathian region near Uzhgorod city was described.

Key words: land molluscs, introduced species, Tandonia cristata, Tandonia kaleniczenkoi, Transcarpathian region

\section{Einleitung}

Das natürliche Verbreitungsgebiet der Nacktschnecke Tandonia kusceri (Wagner, 1931) liegt auf der Balkanhalbinsel, wo sie in Bulgarien, Serbien, im europäischen Teil der Türkei, im Südosten Rumäniens (Dobrogea) (LIKHAREV \& WiKTOR 1980) sowie in Mazedonien, Kroatien (WIKTOR \& JuRKowsKa 2007) und Montenegro (TelebaK et al. 2013) vorkommt. Laut WiKTOR (1996) umfasst das natürliche Areal von T. kusceri auf dem Gebiet des ehemaligen Jugoslawiens lediglich Serbien und Mazedonien. Das Vorkommen dieser Art in anderen Ländern (Kroatien, Montenegro) könnte also auf Anthropochorie zurückzuführen sein. Tandonia kusceri wurde auch in andere europäische Länder (BALASHOV et al. 2013, KORÁBEK et al. 2016) sowie in Nordamerika (GERBER 2014) eingeschleppt. Dies wurde durch die hohe ökologische Plastizität dieser Art und ihre Neigung zur Synanthropie (WIKTOR 1987) erleichtert.

In der Ukraine war T. kusceri lange Zeit ausschließlich aus der Stadt Odessa bekannt (Lindholm 1908, LiKHAREV \& WiKTOR 1980, WiKTOR 1987). Erst am Ende des 20. Jahrhunderts wurde diese Art auch im Stadtgebiet von Nikolaev zusammen mit einer anderen eingeschleppten Nacktschneckenart, Limacus maculatus (Kaleniczenko, 1851), nachgewiesen (KramarenKo \& Sverlova 2001). Anfang des 21. Jahrhunderts wurden weitere Angaben über Funde von T. kusceri in den Verwaltungsgebieten 
Odessa (drei Lokalitäten) und Nikolaev (eine Lokalität) (Son 2004) sowie in der Krim nordwestlich von Simferopol (eine Lokalität) (LEONOV 2007) publiziert. Alle erwähnten Funde weisen deutlich auf eine allmähliche und mit Anthropochorie verbundene Erweiterung des Verbreitungsgebiets von T. kusceri hin.

Im Herbst 2018 wurde T. kusceri erstmals in der Westukraine, nämlich im Süden Transkarpatiens, gefunden. Dieser Fundort ist von besonderem Interesse, da er sowohl vom natürlichen Verbreitungsgebiet der Art (WeLTER-SCHULTES 2012), als auch von deren auf Einschleppung basierenden Vorkommen in der Südukraine entfernt ist. Für die Westukraine war bisher nur ein einziger Vertreter der Familie Milacidae, Tandonia cristata (Kaleniczenko, 1851), bekannt (LIKHAREV \& WiKTOR 1980).

Der Fund von T. kusceri in Transkarpatien veranlasste uns zu einer genaueren Analyse der Verbreitung der Gattung Tandonia Lessona \& Pollonera, 1882 in der Ukraine, da bisher nur wenige exakte Angaben zu den Fundorten dieser Arten vorlagen (BALASHOV 2016).

\section{Material und Methoden}

Das äußere Erscheinungsbild sowie die Genitalien wurden bei drei Individuen von T. kusceri aus dem Verwaltungsgebiet Transkarpatien untersucht. Diese Exemplare wurden von V. Gleba im November 2018 am Rande der Siedlung Korolevo, Bezirk Vinogradov gesammelt (GPS-Koordinaten: $48^{\circ} 08^{\prime} 46.69^{\prime \prime} \mathrm{N} 23^{\circ} 07^{\prime} 55.52^{\prime \prime} \mathrm{E}$ ). Der Fundort befindet sich neben einem Eisenbahndepot, wo Diesel-Personenzüge repariert und gewartet werden. Im Oktober und November 2018 wurden von V. Gleba an derselben Stelle weitere Individuen dieser Art beobachtet, was auf eine etablierte Kolonie von T. kusceri hinweisen dürfte.

Der Fundort wird seit ca. 10 Jahren von der lokalen Bevölkerung als nicht genehmigte Deponie, vor allem für Bauschutt (Ziegelsteine, Glasscherben, Schieferbruchstücke usw.) genutzt. Daneben liegt die schmale, zum Depot führende Zufahrtsstraße und dahinter eine Eisenbahnlinie. Die Nacktschnecken wurden in $70 \%$ Ethanol fixiert und nach der Methodik von LIKHAREv \& WIKTOR 1980 seziert. Für die Determination wurden die Monografien von WIKTOR $(1987,1996)$ verwendet. Die untersuchten Exemplare wurden an die Landschnecken-Sammlung des Schmalhausen-Instituts für Zoologie (Kiev) übergeben (IZAN GT 7139).

Zusätzlich werden publizierte Funddaten verschiedener Tandonia-Arten auf dem Territorium der Ukraine aus eigenen Untersuchungen (KRAMARENKo \& Sverlova 2001, GURAL-SvERLOVA 2018, GURAL-SvERLOVA et al. 2018) sowie jenen anderer Forscher (KaLENICZENKo 1851, ClesSIN 1883, Retowski 1883, Lindholm 1908, ARUTYUNOVA, 1975, LikHAREV \& WikToR 1980, Son 2004, LeONOV 2007, Balashov \& Palatov 2011, Balashov \& BaidasHNIKOV 2012) zusammengefasst und analysiert. Außerdem wurden einige persönliche Mitteilungen von I. Balashov (Schmalhausen-Institut für Zoologie, Kiev, Ukraine) berücksichtigt. Die Fundorte der in der Ukraine vorkommenden Tandonia-Arten wurden in einer Karte dargestellt und die Besonderheiten ihrer heutigen Verbreitung diskutiert. Neben T. kusceri aus Transkarpatien wurden von uns die
Exemplare der Art aus der Südukraine (Odessa, Nikolaev), T. cristata aus der Steppenkrim (Chernomorskoye), aus den Verwaltungsgebieten Odessa (Vilkovo) und Zaporozhye (Vasilyevka) sowie T. kaleniczenkoi aus der Bergkrim (Simferopol) zu verschiedenen Zeiten anatomisch untersucht. Ausführlichere Angaben zu den Fundorten sowie Sammlungszeiten dieses Materials zeigt Tabelle 1.

\section{Ergebnisse und Diskussion}

Die Körpergrundfarbe der Individuen von T. kusceri aus Transkarpatien reicht von dunkelcremefarbig bis hellbraun. Bei lebenden Tieren fällt bei einer gewissen Beleuchtung eine rosa Tönung auf. Das mit zahlreichen kleinen dunklen Flecken gebildete Muster ist auf dem Mantel und Rücken stärker entwickelt. Die Seiten sind heller, die dunkle Pigmentierung, die hauptsächlich entlang der Furchen angeordnet ist, nimmt zum Sohlenrand allmählich ab. Die Flecken sind auf dem Mantel teilweise miteinander verschmolzen und bilden zwei Seitenbänder mit etwas undeutlichen Konturen. Das rechte Band verläuft oberhalb des Pneumostoms, es dabei nicht umfassend, sondern nur leicht von oben berührend (Abb. 1C, D). Eine weitere Anhäufung von dunklen Flecken liegt in der Mantelmitte (Abb. 1D). Mit zunehmendem Alter nimmt die dunkle Pigmentierung wahrscheinlich zu. Mantel und Rücken bei der größten von uns untersuchten Nacktschnecke färbten sich nach der Fixierung fast komplett schwarz (Abb. 1C). Die Farbe des Kiels stimmt mit der Grundfarbe des Körpers überein, erscheint jedoch aufgrund des Fehlens von dunklen Flecken heller. Die Sohle ist einfarbig hell, nach der Fixierung gelblich-cremefarbig. Bei lebenden Nacktschnecken ist eine gelbe Färbung der Sohle noch stärker ausgeprägt (Abb. 1B). Der Schleim ist farblos. Die Färbung von T. kusceri wird in den Monografien von LIKHAREV \& WiKTOR (1980) und WiKTOR (1987) ähnlich beschrieben und abgebildet. Sie stimmt auch gut mit der Färbung der Exemplare von T. kusceri aus der Südukraine (Odessa) überein, die von N. Gural-Sverlova 1999 und 2000 beobachtet and anatomisch untersucht wurden (Abb. 1E).

Die Körperlänge der Nacktschnecken betrug nach der Fixierung 30 bis $47 \mathrm{~mm}$ (Abb. 1C). Ausgestreckt erreichte der Körper des größten Individuums bis $80 \mathrm{~mm}$ Gesamtlänge. Die Körperlänge erwachsener Exemplare von T. kusceri beträgt Literaturangaben zufolge (LIKHAREV \& WiKTOR 1980, WiKTOR 1987) bis $100 \mathrm{~mm}$ ausgestreckt und $65 \mathrm{~mm}$ bei Kontraktion.

Die anatomisch untersuchten Exemplare aus Transkarpatien weisen einen langen Epiphallus auf, der scharfe Kurven bildet (Abb. 2). Dieses Merkmal ist besonders charakteristisch für T. kusceri. Bei anderen bekannten Vertretern der Gattung Tandonia (WIKTOR 1987, 1996, De MatTiA \& NARDI 2014) ist der Epiphallus deutlich kürzer. Nur eine äußerlich and anatomisch ähnliche Art, Tandonia rustica (Millet, 1843), hat einen relativ langen und schmalen Epiphallus, der aber keine solchen scharfen Spiralkurven zeigt. Es gibt auch andere Unterschiede zwischen beiden Arten: T. rustica hat eine längliche, am Ende zugespitzte Spermatheka (Bursa copulatrix) mit einem kurzen Bursastiel (LikHAREV \& WiKTOR 1980, WiKTOR 1987), T. kusceri hingegen eine kugelförmige Spermatheka mit 

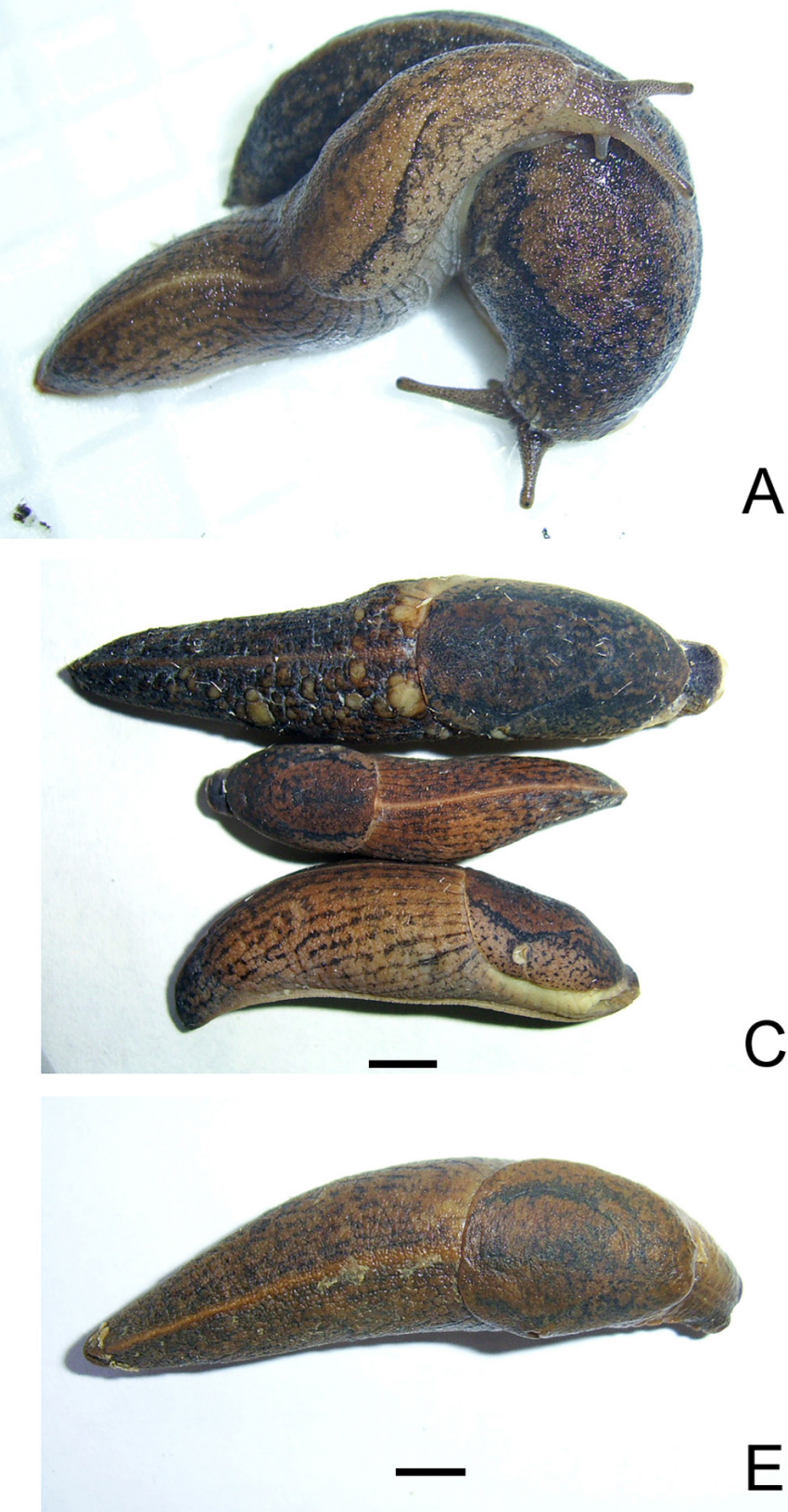

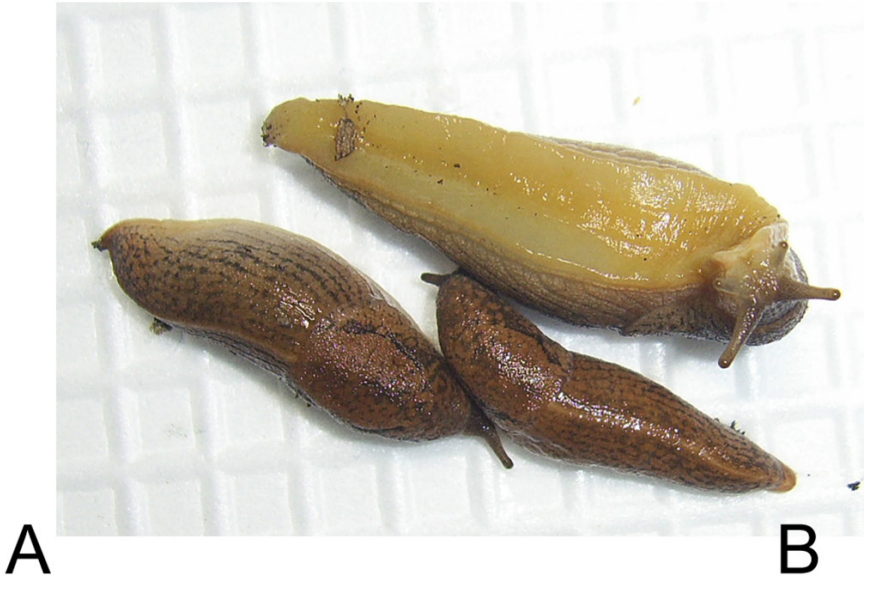

A

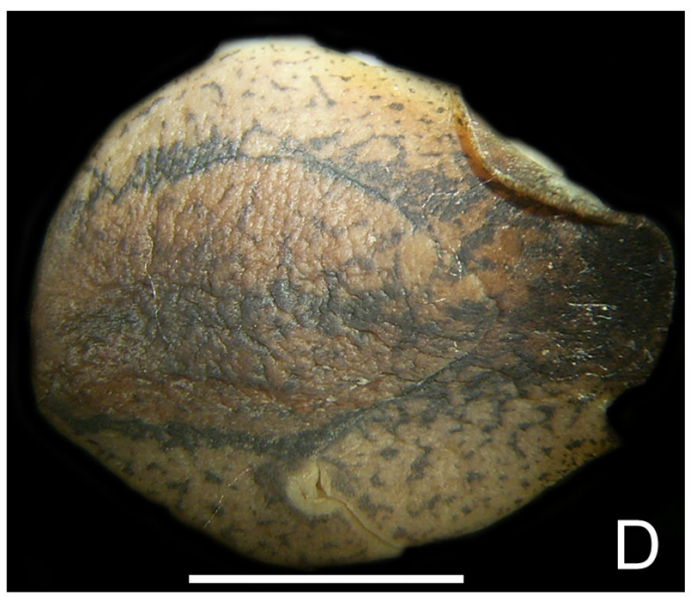

C

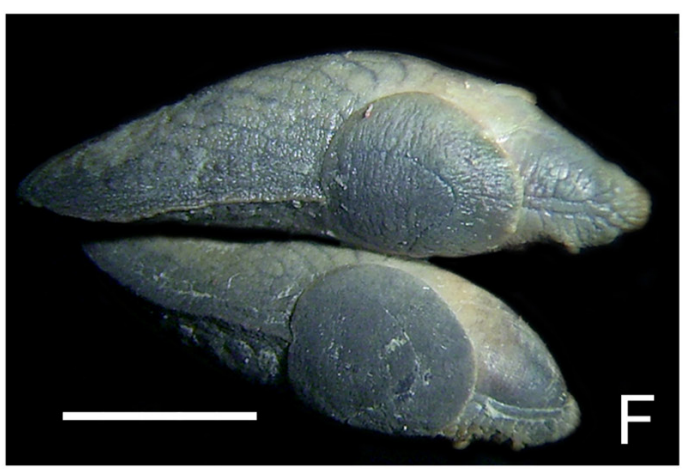

Abb. 1. Äußeres Aussehen von Tandonia kusceri aus Korolevo, Transkarpatien (A-D) und Odessa (E), Tandonia cristata aus Chernomorskoye, Steppenkrim (F); Maßstab $5 \mathrm{~mm}$. Alle Fotos von N. Gural-Sverlova.

Fig. 1. Appearance of Tandonia kusceri from Korolevo, Transcarpathian region (A-D) and Odessa (E), Tandonia cristata from Chernomorskoye, Steppe Crimea (F); scale bars $5 \mathrm{~mm}$. All photos by N. Gural-Sverlova.

einem langen Bursastiel (siehe Abb 2).

Eine Einschleppung von T. kusceri nach Transkarpatien aus anderen europäischen Ländern ist theoretisch nicht auszuschließen. Man dürfte aber die Südukraine als die wahrscheinlichste Quelle solcher Einschleppung annehmen, wo diese Art vereinzelt in Siedlungen und deren Umgebungen vorkommt (Abb. 3). Das Auftreten von T. kusceri in Korolevo könnte mit dem Eisenbahnverkehr verbunden sein, da der Sammelort in unmittelbarer Nähe zum Lokomotivendepot liegt. Zu erwähnen ist, dass nahe den Eisenbahnstationen in Korolevo und den benachbarten Vinogradov zwei große Kolonien einer anderen eingeschleppten Landschneckenart vor kurzem nachgewiesen wurden (GURAL-SVERLOva \& GURAL 2017), und zwar die Steppenart Xeropicta derbentina (Krynicki, 1836), welche heute im Süden der Ukraine weit verbreitet ist (Gural-Sverlova \& Gural 2017, Gural-Sverlova 2018).

T. kusceri ist der zweite Vertreter der Familie Milacidae, der im Verwaltungsgebiet Transkarpatien nachgewiesen ist. Der bisher einzige Fund von Tandonia cristata (Kaleniczenko, 1851) dort ist aus einem Buchenwald bei Uzhgorod bekannt (LIKHAREV \& WIKTOR 1980). Später fand man in der Westukraine aber weder T. cristata noch andere Tandonia-Arten.

Das natürliche Areal von T. cristata umfasst Bulgarien und wahrscheinlich auch den Südosten Rumäniens (Dobrogea) sowie die nördliche Türkei (WiKTOR 1987). In der Ukraine kommt diese Art vor allem in der Bergkrim vor, wo sich auch der Typenfundort von $T$. cristata befindet 


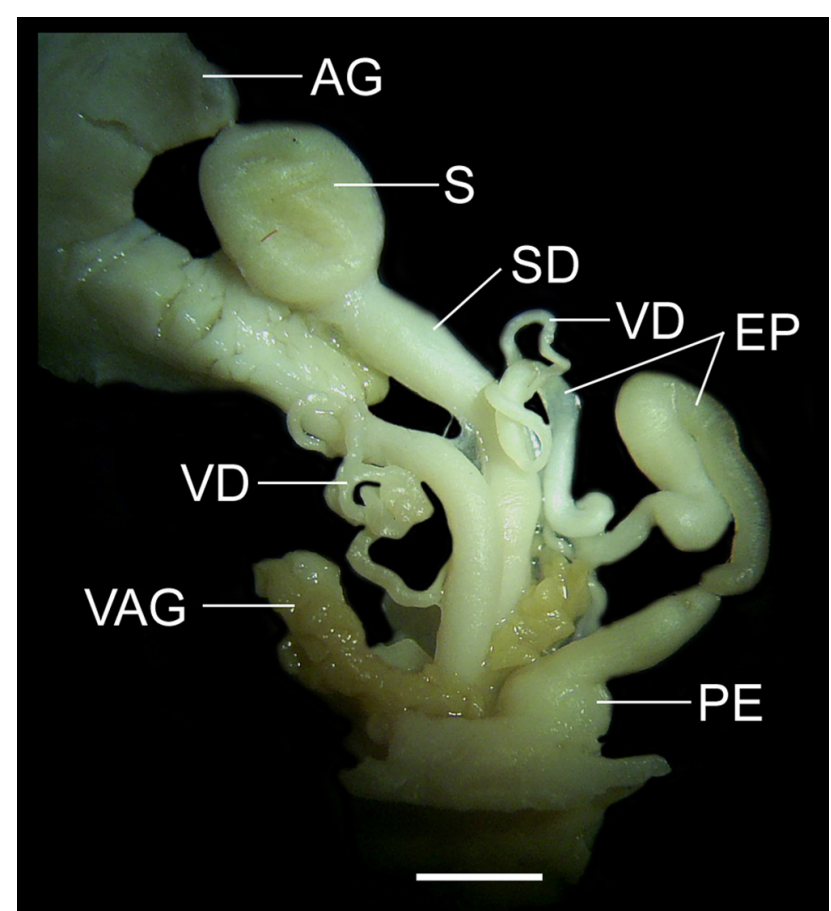

Abb. 2. Genitalien von Tandonia kusceri aus Korolevo, Transkarpatien; Maßstab $2 \mathrm{~mm}$. AG - Eiweißdrüse; EP - Epiphallus; PE - Penis; S - Spermatheka (Bursa copulatrix); SD - Spermathekadukt (Bursastiel); VAG - vaginale Anhangsdrüsen; VD - Samengang.

Fig. 2. Genitalia of Tandonia kusceri from Korolevo, Transcarpathian region; scale bars $2 \mathrm{~mm}$. AG - albumen gland; EP epiphallus; PE - penis; $\mathrm{S}$ - spermatheca (bursa copulatrix); SD spermathecal duct (bursa duct); VAG - vaginal accessory glands; VD - vas deferens.

(KALENiCZENKo 1851). Vor kurzem wurde diese Nacktschnecke auch in der Steppenkrim (Abb. 1F, Tab. 1) registriert. Außerdem sind vereinzelte Funde von T. cristata in der Steppenzone des kontinentalen Teils der Ukraine bekannt (Abb. 3). Bisher besteht kein Konsens darüber, ob die Krim und die nördliche Schwarzmeerküste als Teil des natürlichen Verbreitungsgebiets von T. cristata zu betrachten sind (BALASHOV 2016). Ansonsten könnte diese Art von Menschen hierher eingeschleppt worden sein (WIKTOR 1987), denn sie kommt dort jetzt hauptsächlich synanthrop vor (WIKTOR 1987, BALASHOV 2016).

Der einzige Fund von T. cristata in Transkarpatien (LIKHAREV \& WIKTOR 1980) ist dagegen zweifellos auf eine Einschleppung zurückzuführen, da diese Region weit von allen Teilen ihres Hauptareals (Welter-Schultes, 2012) entfernt ist. Es sei besonders erwähnt, dass in derselben Gegend und möglicherweise auch zusammen mit T. cristata eine andere für die Ukraine anthropochore Nacktschneckenart, Arion distinctus Mabille, 1868, gesammelt wurde (Gural-Sverlova \& Gural 2016), die zunächst als Arion hortensis Férussac, 1819 falsch identifiziert wurde (LikHAREV \& WikTOR 1980). Arion distinctus kommt auch heute im Stadtgebiet von Uzhgorod vor (GURAL-SVERLOVA \& GURAL 2016).

Im Gegensatz zu T. cristata, bezweifelt kein Forscher einen anthropochoren Ursprung aller in der Ukraine registrierten Kolonien von T. kusceri (LIKHAREV \& WIKTOR 1980, Son 2004, Balashov 2016). Diese Art war zuerst in der Hafenstadt Odessa eingeschleppt, was spätestens am Ende des 19. Jahrhunderts geschehen sein soll. Im April 1902 hatte A. Brauner am Langeronplatz in Odessa acht erwachsene Individuen einer großen Nacktschneckenart gesammelt, die später als eine neue Art von LinDHOLM (1908) beschrieben wurde. Obwohl WiKTOR (1987) Amalia rossica Lindholm, 1908 in die Liste der Synonyme von T. kusceri nicht aufnahm, hat er doch die Möglichkeit der Identität dieser Taxa nicht ausgeschlossen (LIKHAREV \& WIKTOR 1980).

LindHolm (1908) hat $A$. rossica nicht anatomisch untersucht, jedoch eine ausführliche Beschreibung des äußeren Aussehens und insbesondere der Körperfärbung von Nacktschnecken gegeben. Er erwähnte dabei einen langen Kiel, der über den ganzen Rücken geht: „Tier... mit deutlich markiertem Rückenkiel, welcher am Mantelrand beginnt und am Hinterende des Körpers scharf vorspringt“". Dies lässt keinen Zweifel daran, dass der Autor einen Vertreter der Familie Milacidae und nicht z.B. eine der in der Ukraine lebenden großen Arten von Limacidae beschrieben hat. Der zweite Teil der Beschreibung behandelt die Körperfärbung von Nacktschnecken, die als sehr typisch für T. kusceri zu betrachten ist: „Kopf hellgrau, Augenträger dunkelgrau; Oberseite hellbräunlich, in der Längsrichtung (d.h. längs der Rinnen) fein schwärzlich gestreift und gestrichelt; der Mantel auf hellbräunlichem Grunde schwärzlich gesprenkelt und punktirt, jederseits mit je einer deutlichen schwärzlichen Längsbinde, zuweilen auch mit einer verschwommenen Mittelbinde; Kiel heller als die Grundfarbe, etwa schmutziggelblich, einfarbig. Unterseite gleichfalls gelblich einfarbig“".

Nur die Zahl der Runzeln zwischen dem Kiel und dem Pneumostom stimmt in den Beschreibungen beider Taxa nicht ganz überein: 9 bis 13 bei $A$. rossica (LINDHOLM 1908) und 14 oder 15 bei T. kusceri (WIKTOR 1987). Dies dürfte aber keinen bedeutenden Unterschied darstellen. Die maximale Länge der mit Ethanol fixierten Exemplare von $A$. rossica betrug $58 \mathrm{~mm}$ (LindHolm 1908). Bei erwachsenen Individuen von T. kusceri kann die Körperlänge bei Kontraktion bis zu $65 \mathrm{~mm}$ erreichen (LIKHAREV \& WiKTOR 1980, WiKTOR 1987).

Der oben erwähnten und mit T. kusceri fast vollkommen übereinstimmenden Beschreibung von $A$. rossica ungeachtet, glauben einige Forscher (LEONOv 2007) aber weiterhin, dass T. kusceri in Odessa zum ersten Mal erst 1939 und zum zweiten Mal 1968 registriert wurde. In beiden Fällen handelt es sich um Exemplare aus der Sammlung des Zoologischen Instituts der Russischen Akademie der Wissenschaften in St. Petersburg, die in einer Parkanlage sowie im Botanischen Garten Odessa gesammelt wurden (LIKHAREV \& WiKTOR 1980).

Bis heute kommt T. kusceri in Odessa vor, wo sie einer der auffallenden Vertreter der städtischen Landschneckenfauna ist (Sverlova et al. 2006). Die anderen Kolonien dieser Art, die am Ende des 20. bzw. am Anfang des 21. Jahrhunderts in den Verwaltungsgebieten Odessa (Son 2004) und Nikolaev (KramarenKo \& Sverlova 2001, Son 2004) im Süden der Ukraine gefunden wurden, dürften von aus Odessa eingeschleppten Exemplaren abstammen. Dies könnte auch für eine Kolonie von T. kusceri gelten, welche 


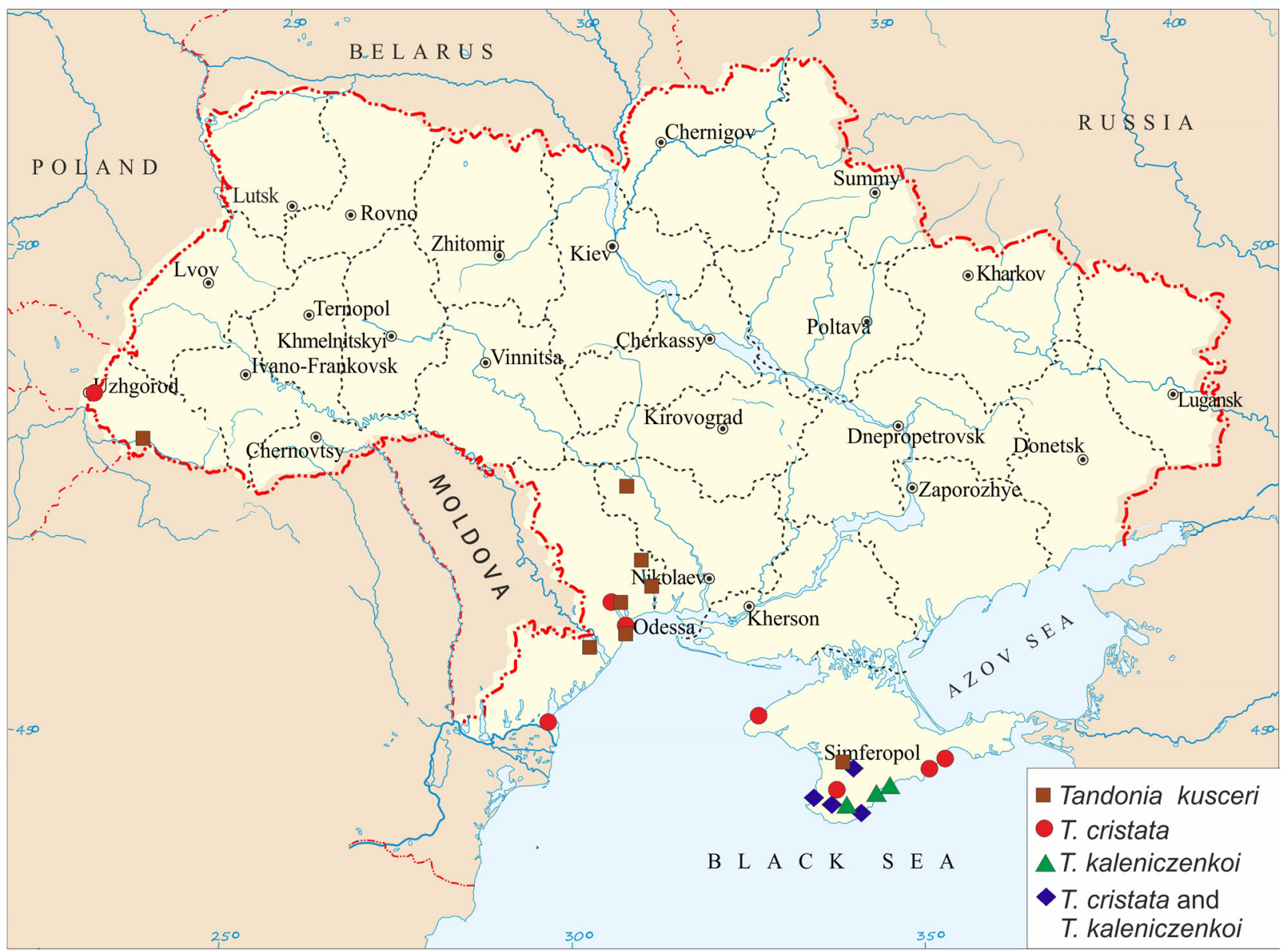

Abb. 3. Fundorte von Tandonia-Arten in der Ukraine.

Fig. 3. Records of Tandonia species in Ukraine.

vor kurzem in der Krim, unweit von Simferopol registriert wurde (LEONOV 2007).

Die dritte in der Ukraine lebende Art der Gattung Tandonia ist Tandonia kaleniczenkoi (Clessin, 1883). In der modernen malakologischen Literatur (WELTER-SCHULTES 2012) wird diese Art auch als Tandonia retowskii (Boettger, 1882) erwähnt. Die Internationale Kommission für Zoologische Nomenklatur hat allerdings kürzlich beschlossen, den Namen T. kaleniczenkoi für diese Art zu behalten (OPINION 2385 (CASE 3642) 2017).

In der Ukraine ist T. kaleniczenkoi ausschließlich in der Bergkrim verbreitet. Nicht selten kommt sie an denselben Orten wie T. cristata vor (Abb. 3, Tab. 1). T. kaleniczenkoi wurde sowohl in natürlichen von Menschen nur wenig gestörten Biotopen (BALASHOV 2016) als auch in menschlichen Siedlungen (BALASHOv \& BaIDASHNiKov 2012) registriert. Letzteres ist durch das von uns selbst untersuchte Material aus Simferopol bestätigt, welches 2006 von V. Martynov auf dem Territorium der Stadt gesammelt wurde.

Obwohl T. kaleniczenkoi in großen und von Touristen oft besuchten Städten der Krim wie Simferopol, Sevastopol und Yalta (LIKHAREV \& WIKTOR 1980) lebt, ist bisher weder eine Einschleppung dieser Nacktschneckenart in andere Regionen der Ukraine noch nach der Steppenkrim bekannt. Außerhalb der Ukraine kommt T. kaleniczenkoi im Südosten Rumäniens (Dobrogea), von wo sie als Milax dobrogicus Grossu, 1982 beschrieben worden war (WIK-
TOR 1987), und in der nördlichen Türkei, im Pontischen Gebirge (LiKHAREv \& WiKTOR 1980, WiKTOR 1987), vor. In einer früheren Publikation von SimRoTH (1886) gibt es die einzige bekannte Erwähnung eines Vorkommens in Sevastopol Amalia gracilis (Leydig, 1876), die man jetzt als Synonym von Tandonia budapestensis (Hazay, 1881) betrachtet (WIKTOR 1987). Nach LiKHAREV \& WiKTOR (1980) dürfte dieser Nachweis an einer Fehlbestimmung liegen, denn in seinen weiteren Publikationen gab der Autor A. gracilis weder für die Krim noch generell für das Russische Reich an. LEONOV (2007) vermutet, dass sich die Mitteilung von Simroth (1886) nicht auf T. budapestensis, sondern auf T. kusceri beziehen könnte. T. kusceri wurde jedoch erst am Anfang des 21. Jahrhunderts auf der Krim festgestellt (siehe oben). Bis dato wurde diese Art weder in Sevastopol noch in seiner Umgebung gefunden. Es ist nicht auszuschließen, dass ein großes Exemplar (bzw. Exemplare) der in der Bergkrim verbreiteten und für Sevastopol bekannten (LIKHAREV \& WIKTOR 1980) Nacktschneckenart T. kaleniczenkoi falsch als A. gracilis identifiziert worden war. T. kaleniczenkoi hat eine ähnliche Körperfärbung, ist aber kleiner als $T$. budapestensis.

\section{Danksagung}

Wir möchten uns bei Dr. Igor Balashov (Schmalhausen-Institut für Zoologie, Kiev) für zusätzliche Informationen über Funde von T. cristata und T. kaleniczenkoi in 
der Ukraine bedanken. Vielen Dank auch an Dr. Michael Son (Institut für Meeresbiologie, Odessa), Dr. Victor Busel (Nationaler Naturpark „Velykyj Lug“, Verwaltungsgebiet Zaporozhye) und Dr. Vladimir Martynov (Nationale Universität Donetsk, später Botanischer Garten Donetsk) für das zu unserer Verfügung gestellte Material aus den Verwaltungsgebieten Odessa und Zaporozhye sowie aus der Krim. Ein besonderer Dank gilt Dr. Heike Reise (Senckenberg Museum für Naturkunde Görlitz) und Dr. Michael Duda (Naturhistorisches Museum Wien) für wertvolle Ratschläge und stilistische Korrekturen am Manuskript.

\section{Literatur}

ARUtyunova L. D., 1975: Заметка о некоторых наземных моллюсках южнобережья Крыма [A note on some land molluscs of the southern coast of Crimea]. - Biological Journal of Armenia, 28(10): 104-109. (in Russian).

BALASHOV I. A., 2016: Охрана наземных моллюсков Украины [Conservation of terrestrial molluscs in Ukraine]. - I. I. Schmalhausen Institute of Zoology, Kiev, 272 pp. (in Russian).

Balashov I. A. \& Baidashnikov A. A., 2012: The first findings of a slug Boettgerilla pallens (Stylommatophora, Boettgerillidae) in Crimea. - Ruthenica, Russian Malacological Journal, 22(2): 111-114.

Balashov I. A. \& Palatov D. M., 2011: Первая находка Vertigo moulinsiana (Gastropoda, Pulmonata) для Украины на территории Крыма [First record of Vertigo moulinsiana (Gastropoda, Pulmonata) for Ukraine on territory of the Crimea]. Vestnik zoologii, 45(1): 11-17. (in Russian).

Balashov I. A., Son M. O., Coadă V. \& Welter-Schultes F., 2013: An updated annotated checklist of the molluscs of the Republic of Moldova. - Folia Malacologica, 21(3): 175-181.

Clessin S., 1883: Anhang zur Molluskenfauna der Krim. - Malakozoologische Blätter. Neue Folge, 6: 37-52.

De Mattia W. \& Nardi G., 2014: Tandonia bolensis n. sp., a new slug (Gastropoda: Pulmonata: Milacidae) from the island of Brač (Dalmatia, Croatia). - Ecologica Montenegrina, 1(2): $123-130$.

Gerber J., 2014: First American record of the exotic slug Tandonia kusceri (Gastropoda: Milacidae). - The Nautilus, 128(2): 59-63.

Gural-Sverlova N. V., 2018: Пространственное распределение наземной малакофауны степной зонь Украины [Spatial distribution of land molluscs fauna of the steppe zone of Ukraine]. - Ruthenica, Russian Malacological Journal, 28(4): 131-138. (in Russian).

Gural-Sverlova N. V., Busel V. A. \& Gural R. I., 2018: Видовой состав наземных моллюсков Запорожской области и влияние на него антропохории [Species composition of land molluscs of Zaporozhye region and anthropochory influence on it]. - Ruthenica, Russian Malacological Journal, 28(3): 101-112. (in Russian).

GuRal-Sverlova N. V. \& Gural R. I., 2016: Новые находки слизней Arion distinctus и Arion circumscriptus (Arionidae) на территории Украины [New findings of the slugs Arion distinctus and Arion circumscriptus (Arionidae) in the territory of Ukraine]. - Ruthenica, Russian Malacological Journal, 26(1): 17-23. (in Russian).

Gural-Sverlova N. V. \& Gural R. I., 2017: Expansion of the ranges of land mollusks of the genus Xeropicta (Gastropoda, Hygromiidae) in Ukraine. - Russian Journal of Biological Invasions, 8(3): 212-217.
Kaleniczenko J., 1851. Description d'un nouveau genre de Limaces de la Russie meridionale. - Bulletin de la Société impériale des naturalistes de Moscou, 24(1): 215-228.

KoRÁBEK O., ČEJKA T. \& JUŘIČKOVÁ L., 2016: Tandonia kusceri (Pulmonata: Milacidae), a slug new for Slovakia. - Malacologica Bohemoslovaca, 15: 3-8.

Kramarenko S. S. \& Sverlova N. V., 2001: Наземная малакофауна (Gastropoda, Pulmonata) Николаевской области [Land molluscs fauna (Gastropoda, Pulmonata) of Nikolaev region]. - Vestnik zoologii, 35(2): 75-78. (in Russian).

LeONov S. V., 2007: The first record of the Tandonia kusceri (Pulmonata, Milacidae) in Crimea and a few remarks about its natural habitats. - Vestnik zoologii, 41(2): 142.

Likharev I. M. \& WiкToR A. J., 1980: Слизни фауны СССР и сопредельных стран (Gastropoda terrestria nuda) [Slugs from the fauna of the USSR and neighbouring countries (Gastropoda terrestria nuda)]. - Fauna of the USSR. New series, 122. Molluscs, 3(5). Nauka, Leningrad, 438 pp.

Lindholm W. A., 1908: Materialien zur Molluskenfauna von Südwestrussland, Polen und der Krim. - Записки Новороссийского общества естествоиспытателей [Proceedings of the Novorossiysk Natural History Society], 31: 199-232.

OPINION 2385 (CASE 3642), 2017: Amalia kaleniczenkoi Clessin, 1883 (Gastropoda, Stylommatophora, Milacidae): specific name conserved. - The Bulletin of Zoological Nomenclature, 73(2-4): 154-155.

Retowski O., 1883: Die Molluskenfauna der Krim. - Malakozoologische Blätter. Neue Folge, 6: 1-34.

Simroth H., 1886: Ueber bekannte und neue palaearktische Nacktschnecken. - Jahrbücher der Deutschen Malakozoologischen Gesellschaft, 13: 311-342.

Son M. O., 2004: Новые находки Tandonia kusceri (Pulmonata, Milacidae) в Украине [New records of Tandonia kusceri (Pulmonata, Milacidae) in Ukraine]. - Vestnik zoologii, 38(3): 76. (in Russian).

Sverlova N. V., Khlus L. N., Kramarenko S. S., Son M. O., Leonov S. V., Korol E. N., Vychalkovskaya N. V., Zemoglyadchuk K. V., Kyrpan S. P., Kuzmovych M. L., Stenko R. P., Ferenz O. G., Shklyaruk A. N. \& Gural R. I., 2006: Фауна, экология и внутривидовое разнообразие наземных моллюсков в урбанизированной среде [Fauna, ecology and intraspecific variability of land molluses under urban conditions]. - State Museum of Natural History, Lviv, 226 pp. (in Russian).

TelebaK B., Brajković M. \& Ćurčić S., 2013: Contribution to the knowledge of the slugs (Gastropoda: Stylommatophora: Limacidae and Milacidae) from Montenegro. - Bulletin of the Natural History Museum in Belgrade, 6: 55-64.

Welter-Schultes F., 2012: European non-marine molluscs, a guide for species identification. - Planet Poster Editions, Göttingen, $679 \mathrm{pp}$.

WikTor A., 1987: Milacidae (Gastropoda, Pulmonata) - systematic monograph. - Annales Zoologici, 41(3): 153-319.

WiKtor A., 1996: The slugs of the former Yugoslavia (Gastropoda terrestria nuda - Arionidae, Milacidae, Limacidae, Agriolimacidae). - Annales Zoologici, 46 (1-2): 1-110.

WikToR A. \& JURKowsKa J., 2007: The collection of terrestrial slugs (Gastropoda: Pulmonata) at the Museum of Natural History, Wrocław University (Poland). - Folia Malacologica, 15: 83-93. 
Tabelle 1. Fundorte von Tandonia-Arten in der Ukraine (IZAN GT- Inventarnummern in der Sammlung von Landmollusken des Schmalhausen-Instituts für Zoologie, Kiev, nach persönliche Mitteilung von I. Balashov).

Table 1. Localities of Tandonia species in Ukraine (IZAN GT- Inventory numbers in the collection of land molluscs of the Schmalhausen Institute of Zoology, Kiev, according to personal communication by I. Balashov).

\begin{tabular}{|c|c|c|c|c|}
\hline \multicolumn{2}{|c|}{$\begin{array}{l}\text { Verwaltungseinheiten / Administrative } \\
\text { divisions }\end{array}$} & \multirow{2}{*}{$\begin{array}{l}\text { Siedlungen bzw. } \\
\text { ihre Umgebungen } \\
\text { / Settlements or } \\
\text { their vicinities }\end{array}$} & \multirow{2}{*}{$\begin{array}{l}\text { Sammlungsja- } \\
\text { hre / Collecti- } \\
\text { on years }\end{array}$} & \multirow[t]{2}{*}{ Quellen / Sources } \\
\hline Gebiete / Regions & Bezirke / Districts & & & \\
\hline \multicolumn{5}{|c|}{ Tandonia kusceri (Wagner, 1931) } \\
\hline \multirow[t]{7}{*}{ Odessa } & \multirow[t]{3}{*}{-} & \multirow[t]{3}{*}{ Stadt Odessa } & 1902 & $\begin{array}{l}\text { LINDHOLM } 1908 \text { als Amalia rossi- } \\
c a \text {, locus typicus }\end{array}$ \\
\hline & & & 1939 und 1968 & LIKHAREV \& WIKTOR 1980 \\
\hline & & & $\begin{array}{l}\text { zahlreiche } \\
\text { Funde am } \\
\text { Ende des } 20 . \\
\text { und am Beginn } \\
\text { des 21. Jahr- } \\
\text { hunderts }\end{array}$ & $\begin{array}{l}\text { SvERLOVA et al. 2006, Beobachtun- } \\
\text { gen von N. Gural-Sverlova, M. } \\
\text { Son, A. Shklyaruk }\end{array}$ \\
\hline & Belyayevka & Dorf Ilinka & 2001 & Son 2004 \\
\hline & Berezovka & Stadt Berezovka & 2001 & Son 2004 \\
\hline & $\begin{array}{l}\text { Limanskiy (bis } 2016 \\
\text { Kominternovo) }\end{array}$ & Dorf Petrovka & 2002 & Son 2004 \\
\hline & $\begin{array}{l}\text { Belgorod-Dnestrov- } \\
\text { skiy }\end{array}$ & $\begin{array}{l}\text { Dorf Pivdennoye, } \\
\text { am Ufer der } \\
\text { Dnister-Mündung } \\
\text { (Dnister-Liman) }\end{array}$ & 2006 & $\begin{array}{l}\text { coll. M. Son, det. N. Gural-Sverlo- } \\
\text { va, } 1 \text { Expl. }\end{array}$ \\
\hline \multirow[t]{2}{*}{ Nikolaev } & - & Stadt Nikolaev & 1998 & KRAMARENKO \& SVERLOVA 2001 \\
\hline & Pervomaysk & Dorf Krymka & 2001 & Son 2004 \\
\hline Krim & Simferopol & Dorf Beloglinka & 2004 und 2006 & LEONOV 2007 \\
\hline Transkarpatien & Vinogradov & Siedlung Korolevo & 2018 & $\begin{array}{l}\text { coll. V. Gleba, det. N. Gural-Sver- } \\
\text { lova, } 3 \text { Expl., IZAN GT } 7139 \text { (und } \\
\text { weitere Individuen, beobachtet von } \\
\text { V. Gleba) }\end{array}$ \\
\hline \multicolumn{5}{|c|}{ Tandonia cristata (Kaleniczenko, 1851) } \\
\hline \multirow[t]{9}{*}{ Krim (Bergteil) } & \multirow[t]{2}{*}{ Feodosiya } & $\begin{array}{l}\text { Kisiltash beim } \\
\text { Dorf Otuz (jetzt } \\
\text { Siedlung Shche- } \\
\text { betovka), locus } \\
\text { typicus }\end{array}$ & 1837 & KALENICZENKO 1851, tab. 5, fig. 1 \\
\hline & & Stadt Feodosiya & $?$ & RETOWSKI 1883 \\
\hline & \multirow[t]{2}{*}{ Yalta } & \multirow[t]{2}{*}{ Stadt Yalta } & $?$ & RETOWSKI 1883 \\
\hline & & & 1956 & $\begin{array}{l}\text { LIKHAREV \& WIKTOR 1980, fig. } \\
489\end{array}$ \\
\hline & \multirow[t]{3}{*}{ Sevastopol } & $\begin{array}{l}\text { Stadt Sevastopol } \\
\text { (Chersonesus) }\end{array}$ & 1973 & ARUTYUNOVA 1975, fig. 2 \\
\hline & & Stadt Sevastopol & $?$ & LIKHAREV \& WIKTOR 1980 \\
\hline & & $\begin{array}{l}\text { zwischen den Dör- } \\
\text { fern Ternovka und } \\
\text { Khodzha-Sala }\end{array}$ & $2009-2010$ & $\begin{array}{l}\text { BALASHOV \& Palatov 2011, IZAN } \\
\text { GT 1413, 1419, } 7 \text { Expl. }\end{array}$ \\
\hline & Simferopol & Stadt Simferopol & $?$ & LIKHAREV \& WIKTOR 1980 \\
\hline & Bakhchisaray & $\begin{array}{l}\text { Dorf Predushchel- } \\
\text { noye }\end{array}$ & 2009 & $\begin{array}{l}\text { coll. et det. I. Balashov, IZAN GT } \\
\text { 1414, } 1 \text { Expl. }\end{array}$ \\
\hline $\begin{array}{l}\text { Krim (Steppen- } \\
\text { teil) }\end{array}$ & Chernomorskoye & $\begin{array}{l}\text { Siedlung Cherno- } \\
\text { morskoye }\end{array}$ & 2006 & $\begin{array}{l}\text { coll. V. Martynov, det. N. Gural- } \\
\text {-Sverlova, } 3 \text { Expl. }\end{array}$ \\
\hline
\end{tabular}


Tabelle 1. Fortsetzung.

Table 1. Continued.

\begin{tabular}{|c|c|c|c|c|}
\hline \multirow[t]{3}{*}{ Odessa } & - & Stadt Odessa & $?$ & LiKHAREV \& WiKTOR 1980 \\
\hline & Kiliya & Stadt Vilkovo & 2002 & $\begin{array}{l}\text { coll. M. Son, det. N. Gural-Sverlo- } \\
\text { va, } 2 \text { Expl. }\end{array}$ \\
\hline & Razdelnay & Dorf Egorovka & 2010 & $\begin{array}{l}\text { coll. M. Son, det. I. Balashov, } \\
\text { IZAN GT 1710, } 2 \text { Expl. }\end{array}$ \\
\hline Zaporozhye & Vasilyevka & Stadt Vasilyevka & 2017 & GURAL-SVERLOVA et al. 2018 \\
\hline Transkarpatien & - & Stadt Uzhgorod & $?$ & LIKHAREV \& WIKTOR 1980 \\
\hline \multicolumn{5}{|c|}{ Tandonia kaleniczenkoi (Clessin, 1883) } \\
\hline \multirow[t]{11}{*}{ Krim (Bergteil) } & \multirow[t]{4}{*}{ Yalta } & $\begin{array}{l}\text { Strateis (jetzt Teil } \\
\text { von Yalta), locus } \\
\text { typicus }\end{array}$ & $?$ & Clessin 1883, taf. 2, fig. 11 \\
\hline & & \multirow[t]{2}{*}{ Stadt Yalta } & $?$ & LIKHAREV \& WIKTOR 1980 \\
\hline & & & 1987 & BALASHOV \& BAIDASHNIKOV 2012 \\
\hline & & $\begin{array}{l}\text { Botanischer } \\
\text { Garten Nikita bei } \\
\text { Yalta }\end{array}$ & 1956 & $\begin{array}{l}\text { LIKHAREV \& WIKTOR 1980, fig. } \\
497,502-504\end{array}$ \\
\hline & \multirow[t]{2}{*}{ Sevastopol } & Stadt Sevastopol & $?$ & LIKHAREV \& WIKTOR 1980 \\
\hline & & Dorf Ternovka & 2009 & $\begin{array}{l}\text { coll. et det. I. Balashov, IZAN GT } \\
\text { 1417, 1 Expl. } \\
\end{array}$ \\
\hline & \multirow[t]{2}{*}{ Simferopol } & \multirow[t]{2}{*}{ Stadt Simferopol } & $?$ & LIKHAREV \& WIKTOR 1980 \\
\hline & & & 2006 & $\begin{array}{l}\text { coll. V. Martynov, det. N. Gural- } \\
\text {-Sverlova, } 5 \text { Expl. }\end{array}$ \\
\hline & \multirow[t]{2}{*}{ Alushta } & Stadt Alushta & 1987 & BALASHOV \& BAIDASHNIKOV 2012 \\
\hline & & $\begin{array}{l}\text { Siedlung Solnech- } \\
\text { nogorskoye }\end{array}$ & 2008 & $\begin{array}{l}\text { coll. et det. I. Balashov, IZAN GT } \\
\text { 1415, } 3 \text { Expl. }\end{array}$ \\
\hline & Bakhchisaray & Dorf Sokolinoye & 2009 & $\begin{array}{l}\text { coll. et det. I. Balashov, IZAN GT } \\
\text { 1653, } 1 \text { Expl. }\end{array}$ \\
\hline
\end{tabular}

